\title{
Uso de softwares digitais como ferramenta de educação médica no ensino na interpretação do eletrocardiograma: uma revisão sistemática
}

\author{
Use of digital software as a medical education tool in teaching electrocardiogram interpretation: a
} systematic review

Uso de software digital como herramienta de educación médica em la enseñanza de la interpretación de electrocardiogramas: una revisión sistemática

\section{Resumo}

Em 1887 foi registrado o primeiro eletrocardiograma humano e em 1902 invetado o eletrocardiógrafo. Este representa a atividade elétrica cardíaca de forma não invasiva pela superfície do corpo e é componente para avaliação de doenças cardíacas. É de baixo custo e risco. O eletrocardiograma é ferramenta importante e tem interpretação precária por estudantes de medicina e médicos e, dessa forma, novas tecnologias digitais por meio de softwares são usados para facilitar o processo. A educação médica com assistência dessas plataformas digitais permite aprimorar o conhecimento, compreendimento e aprendizado. $\mathrm{O}$ objetivo desta revisão sistemática de literatura é avaliar o ensino na interpretação em eletrocardiograma na área médica e identificar a melhor forma de ensino: tradicional, por meio de aulas presenciais, digital utilizando plataformas tecnológicas ou a combinação de ambas metodologias. Foram avaliados após os critérios de exclusão 12 trabalhos científicos abordando o tempo educação médica em interpretação do eletrocardiograma, utilizando os termos eletrocardiograma, ensino ou aprendizagem, computador ou baseado em web ou software e seus correlatos em inglês. Pode-se concluir, após revisão, que, considerando os estudos avaliados o uso das plataformas digitais proveram aprimoramento nas habilidades de interpretação do eletrocardiograma devido disponibilidade em horários e locais mais flexíveis e repetição conforme necessário. Entretanto, não definição horário específico, necessidade de disciplina por parte do aprendiz e a ausência de interatividade em grupo são fatores que afastam os estudantes deste método e mostrando, que este ensino não deve ser usado de forma isolada e sim de forma complementar ao método tradicional.

Palavras-chave: Eletrocardiograma; Educação médica; Ensino; Estudante; Ensino à distância.

\begin{abstract}
In 1887 the first human electrocardiogram was registered and in 1902 the electrocardiograph was invented. This represents the cardiac electrical activity in a non-invasive way through the surface of the body and is a component for the evaluation of cardiac diseases. It is low cost and risk. The electrocardiogram is an important tool and is poorly interpreted by medical students and physicians and, therefore, new digital technologies through software are used to facilitate the process. Medical education with the assistance of these digital platforms allows to improve knowledge, understanding and learning. The objective of this systematic literature review is to evaluate the teaching of
\end{abstract}


electrocardiogram interpretation in the medical field and to identify the best way of teaching: traditional, through faceto-face classes, digital using technological platforms or a combination of both methodologies. Twelve scientific papers addressing medical education time in electrocardiogram interpretation, using the terms electrocardiogram, teaching or learning, computer or web-based or software and their correlates in English, were evaluated after the exclusion criteria. It can be concluded, after reviewing, that, considering the studies evaluated, the use of digital platforms provided improvement in the skills of interpreting the electrocardiogram due to availability at more flexible times and places and repetition as needed. However, not defining a specific time, the need for discipline on the part of the learner and the absence of group interactivity are factors that distance students from this method and showing that this teaching should not be used in isolation, but in a complementary way to the traditional method.

Keywords: Electrocardiogram; Medical education; Teaching; Students; Distance learning.

\section{Resumen}

En 1887 se registró el primer electrocardiograma humano y en 1902 se inventó el electrocardiógrafo. Representa la actividad eléctrica cardíaca de forma no invasiva a través de la superficie del cuerpo y es un componente para la evaluación de enfermedades cardíacas. Es de bajo costo y riesgo. El electrocardiograma es una herramienta importante y es mal interpretado por estudiantes de medicina y médicos y, por tanto, se utilizan nuevas tecnologías digitales a través de software para facilitar el proceso. La educación médica con la ayuda de estas plataformas digitales permite mejorar el conocimiento, la comprensión y el aprendizaje. El objetivo de esta revisión sistemática de la literatura es evaluar la enseñanza de la interpretación de electrocardiogramas en el campo médico e identificar la mejor forma de enseñanza: tradicional, a través de clases presenciales, digital utilizando plataformas tecnológicas o una combinación de ambas metodologías. Doce artículos científicos que abordan el tiempo de educación médica en la interpretación de electrocardiogramas, utilizando los términos electrocardiograma, enseñanza o aprendizaje, computador o basado en la web o software y sus correlatos en inglés, fueron evaluados después de los criterios de exclusión. Se puede concluir, luego de revisar, que, considerando los estudios evaluados, el uso de plataformas digitales proporcionó una mejora en las habilidades de interpretación del electrocardiograma debido a la disponibilidad en tiempos y lugares más flexibles y repetición según las necesidades. Sin embargo, no definir un tiempo específico, la necesidad de disciplina por parte del aprendiz y la ausencia de interactividad grupal son factores que alejan a los estudiantes de este método y muestran que esta enseñanza no debe utilizarse de forma aislada, sino de forma complementaria a el método tradicional.

Palabras clave: Electrocardiograma; Educación médica; Enseñanza; Estudiantes; Enseñanza a distancia.

\section{Introdução}

Em 1887, o fisiologista Augusttus D. Walker registrou o primeiro eletrocardiograma humano, conectando eletrodos no tórax, anterior e posteriormente e, em 1902 inventado o eletrocardiógrafo pelo fisiologista holandês Willem Einthoven (Giffoni e Torres, 2010). O eletrocardiograma representa a atividade elétrica cardíaca percebida de forma não invasiva pela superfície do corpo e é componente integral da avaliação inicial de doenças cardíacas (Sattar e Chhabra, 2021). É um exame de baixos custo e risco usado para identificar arritmias, isquemia cardíaca, distúrbios hidroeletrolíticos e complicações cardíacas de doenças pulmonares (Pastore et al, 2016).

O ensino de eletrocardiograma para estudantes segue paradigmas de interpretação que seguem uma linha tradicional de aprendizado através de leitura e aulas tradicionais (Pontes et al, 2018). É, nas universidades, ensinada por especialistas para turmas com grande número de estudantes (Cantilon, 2003).

O eletrocardiograma é uma importante ferramenta e tem interpretação precária por estudantes de medicina e médicos, por isso uso de novas tecnologias digitais tem como objetivo facilitar o processo de adquirir conhecimento (Fent et al, 2015). Assim, diversos softwares com foco no ensino sobre eletrocardiograma são criados para computadores, além de outros dispositivos, como tablets e smartphones (Marçal et al, 2005).

Educação médica com assistência de plataformas digitais permite aos aprendizes aprimorar seu conhecimento, compreendimento e aprendizado (Henry, 1990). Proporciona, aos usuários, maior controle sobre a matéria em si, escolha do espaço de aprendizado, domínio do tempo em que será realizado e eficiência ao realizar uma tarefa (Clayden e Wilson, 1988). Os programas podem auxiliar e reduzir o tempo usado para maximizar determinada matéria (Piemme, 1988).

O objetivo dessa revisão sistemática é avaliar, na literatura atual, o ensino da interpretação em eletrocardiograma na 
área médica e identificar a melhor forma de ensino: tradicional, por meio de aulas presenciais, digital utilizando plataformas tecnológicas ou a com combinação de ambas metodologias.

\section{Metodologia}

Essa revisão sistemática baseia-se de acordo na sistemática ao Prefered Reporting Items for Systematic Review (PRISMA), seguindo etapas propostas por Pereira e Galvão (2004): 1. Elaboração da pergunta de pesquisa; 2. Busca na literatura; 3. Seleção dos artigos; 4. Extração dos dados; 5. Avaliação da qualidade metodológica e 6. Síntese dos dados.

Esta sintetiza a literatura em ensino da interpretação do eletrocardiograma em medicina. No período entre 1 de Março de 2021 a 13 de Agosto de 2021, foram analisados artigos por meio das plataformas: Pubmed, Medline, Cochrane, Science Direct e Scielo, com último acesso sendo realizado em 13 de Agosto de 2021. Para a busca dos mesmos, foram-se usados termos como: eletrocardiograma, ensino ou aprendizagem, computador ou baseado em web ou software e seus correlatos em inglês.

Com esses termos foram encontrados 81 artigos e estes foram selecionados conforme os critérios de inclusão e exclusão do protocolo. Os primeiros foram incluídos com base na presença de conteúdo sobre ensino ou aprendizagem na interpretação do eletrocardiograma em torno do ensino tradicional ou utilizando plataformas digitais ou até mesmo com ambas formas de ensino. Foram excluídos aqueles artigos que não citavam sobre ensino de eletrocardiograma e aqueles que não incluíram ensino avaliando o uso de tecnologias digitais. Não foram excluídos artigos baseados em sua linguagem ou data de publicação. No final, 12 artigos sobraram para análise da revisão e suas principais características são detalhadas no Quadro 1.

Quadro 1 - Estudos, ano de publicação, objetivo e achados principais

\begin{tabular}{|c|c|c|c|}
\hline Estudo & Ano & Objetivo & Achados \\
\hline Davies et al & 2016 & $\begin{array}{l}\text { Comparar a eficácia do programa aprendizado } \\
\text { eletrônico com grupos tutoriais na fase pré-clínica do } \\
\text { currículo médico. }\end{array}$ & $\begin{array}{l}\text { O grupo baseado em tutorias demonstrou melhor } \\
\text { performance que o grupo aprendizado eletrônico na } \\
\text { avaliação final. }(84 \% \times 74,5 \%)\end{array}$ \\
\hline Bojsen et al & 2015 & 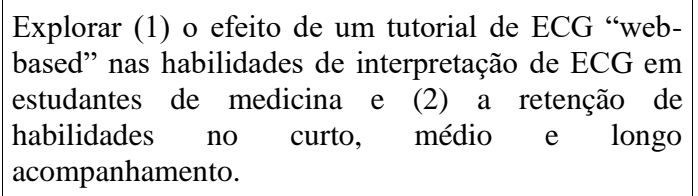 & $\begin{array}{l}\text { Houve melhora significativa do pré-teste para pós- } \\
\text { teste, mas com redução entre o pós-teste e o teste de } \\
\text { retenção. }\end{array}$ \\
\hline Keis et al & 2017 & $\begin{array}{l}\text { Usado o fato que ensino ECG ofertado de dois } \\
\text { formatos diferentes para examinar as seguintes } \\
\text { questões: (1) Quais razões porque estudantes } \\
\text { escolhem método frente a frente ou online se } \\
\text { conteúdo de ambos é idêntico e (2) Que conclusões } \\
\text { são tiradas dessas preferências para criar formatos de } \\
\text { ensino eletrônico que melhor atenda às necessidades } \\
\text { do estudante. }\end{array}$ & $\begin{array}{l}\text { Aulas tradicionais promoveram mais motivação e } \\
\text { eficácia, sugeriram que o online tivesse feedback } \\
\text { pessoal. Preferiram métodos tradicionais com maior } \\
\text { interação com quem ensina e os demais estudantes, } \\
\text { encaixaram a interação como fator chave para o } \\
\text { sucesso do aprendizado. Por isso, forma híbrida de } \\
\text { ensino é o recomendado. }\end{array}$ \\
\hline Viljoen et al & 2020 & $\begin{array}{l}\text { Comparar a efetividade da combinação de aulas } \\
\text { tradicionais (frente a frente) e ensino eletrônico com } \\
\text { ensinamento convencional de ECG (frente a frente } \\
\text { isolada) na aquisição e retenção de competências em } \\
\text { ECG de estudantes de medicina. }\end{array}$ & $\begin{array}{l}\text { Ambas coortes aprimoraram competência após } 6 \\
\text { semanas (maior valor visto no grupo combinado). } \\
\text { Após } 6 \text { meses o grupo ensino tradicional teve queda } \\
\text { significativa para números próximos ao pré-teste, ao } \\
\text { passo que, o grupo combinado, apesar da queda, } \\
\text { manteve retenção de quase duas vezes mais o valor } \\
\text { pré-teste. }\end{array}$ \\
\hline Fent et al & 2016 & $\begin{array}{l}\text { Hipotetizou que estudantes de medicina usando o } \\
\text { "Epicardio Simulation" demonstrariam melhor } \\
\text { habilidade de interpretação de ECG imediatamente } \\
\text { após e } 3 \text { meses após o uso do programa comparados } \\
\text { aqueles que receberam ensinamentos tradicionais }\end{array}$ & $\begin{array}{l}\text { Média de escores foram maiores no grupo tradicional } \\
\text { que no grupo com uso do simulador }(7.07 \times 6.62) \text {. } \\
\text { Não houve diferença no relato próprio de confiança } \\
\text { na interpretação do ECG entre os dois grupos. Após } \\
3 \text { meses não houve diferença significativa entre as } \\
\text { médias dos grupos }(5.79 \times 5.3) \text {. }\end{array}$ \\
\hline
\end{tabular}




\begin{tabular}{|c|c|c|c|}
\hline Fincher et al & 1986 & $\begin{array}{l}\text { Relatar os resultados de um ensaio educacional para } \\
\text { avaliar se o aprendizado com assistência do } \\
\text { computador é mais efetivo que seminários semanais } \\
\text { para ensinar eletrocardiografia fundamental }\end{array}$ & $\begin{array}{l}\text { Avaliado em pré e pós teste com aumento da nota } \\
\text { escolar naqueles do grupo com ensino baseado no } \\
\text { computador }\end{array}$ \\
\hline Barthelemy et al & 2015 & $\begin{array}{l}\text { O alvo do estudo é avaliar a acurácia na interpretação } \\
\text { de eletrocardiogramas clinicamente significantes e } \\
\text { comparar duas estratégias de ensino baseado em } \\
\text { aulas e baseado em ensino eletrônico }\end{array}$ & $\begin{array}{l}\text { Globalmente, houve melhora na média pós teste em } \\
\text { ambos os grupos }\end{array}$ \\
\hline Montassier et al & 2016 & $\begin{array}{l}\text { Comparar a eficiência dos cursos baseado em aulas e } \\
\text { baseado em aprendizado eletrônico para aprimorar } \\
\text { habilidades de interpretação em eletrocardiograma }\end{array}$ & $\begin{array}{l}\text { Comparando os grupos pós-teste o grupo em } \\
\text { aprendizado eletrônico foi não inferior ao baseado } \\
\text { em aulas }\end{array}$ \\
\hline Kopec et al & 2018 & $\begin{array}{l}\text { Comparar a eficiência de duas estratégias de } \\
\text { aprendizado eletrônico no desenvolvimento de } \\
\text { habilidades de interpretação em eletrocardiograma } \\
\text { em estudantes de medicina (colaborativa e } \\
\text { autoaprendizado) }\end{array}$ & $\begin{array}{l}\text { Estudantes do grupo colaborativo foram capazes de } \\
\text { diagnosticar anormalidades mais frequentemente que } \\
\text { aqueles no grupo do autoaprendizado }\end{array}$ \\
\hline Akbarzadeh et al & 2012 & $\begin{array}{l}\text { Avaliar a eficácia da educação baseada em ensino } \\
\text { eletrônico para o eletrocardiograma normal }\end{array}$ & $\begin{array}{l}\text { Educação baseada em ensino eletrônico pode ser tão } \\
\text { eficiente quanto ensino baseado em aulas }\end{array}$ \\
\hline Nilsson et al & 2008 & $\begin{array}{l}\text { Avaliar programa de interpretação de } \\
\text { eletrocardiograma para estudantes de medicina e } \\
\text { avaliar o impacto e efetividade comparando o } \\
\text { aprendizado no grupo intervenção e no grupo } \\
\text { controle }\end{array}$ & $\begin{array}{l}\text { O grupo intervenção no pós teste obteve pontuação } \\
\text { média superior aos controles }(9,7 \times 8,1)\end{array}$ \\
\hline
\end{tabular}

Fonte: Autores (2021).

\section{Resultados e Discussão}

Fincher et al comparou dois grupos de estudantes de medicina, o primeiro com seminários semanais durante 1 hora e o segundo com uso de software de computador e ambos apresentavam o mesmo tema de conceitos básicos em eletrocardiograma. Foi realizado pré teste e pós teste variando de 0 a 10, a média pré teste foi 0.48 e 0.85 respectivamente, pós teste foi 4.91 e 6.58 respectivamente, com média de aumento de 4.36 e 5.69 respectivamente.

Já Akbarzadeh et al avaliou em dois grupos ambos com estudantes de medicina com e sem experiência prévia em eletrocardiograma os conhecimentos pré teste e pós teste acerca do ECG normal, o primeiro consistia no uso de software com assuntos e imagens para ensinar ECG normal e o segundo consistia no ensino por aulas tradicionais em abordagem tanto quantitativa quanto qualitativa. $\mathrm{Na}$ abordagem quantitativa a porcentagem de acertos no pós teste não diferiu entre os grupos ( $72 \%$ e $71 \%$ respectivamente). Na abordagem qualitativa, considerando os estudando sem e com experiência prévia $87 \%$ e $73 \%$ respectivamente acharam o software interessante, considerando os que queriam aprender as porções do ECG pelo software $80 \% 93 \%$ respectivamente e considerando satisfação pelo uso do software $80 \%$ e $60 \%$ respectivamente.

Para Montassier et al comparar a efetividade e a não inferioridade do ensino baseado em mídias digitais (não tinham contato com nenhum tutor e não havia vídeos de tutores com aulas) com ensino em aulas tradicionais para aprimorar habilidades em interpretar ECG em estudantes de medicina. Ambos os grupos foram submetidos a teste antes e 2 semanas após o término de 6 semanas do ensino, o teste compreendeu avaliação em múltipla escolha acerca de 10 eletrocardiogramas. Na avaliação pré teste ambos os grupos tiveram uma média de 9 pontos $(\mathrm{p}=0.9)$. Na avaliação pós teste o grupo mídias digitais foi não inferior ao grupo aulas tradicionais com pontuação 14.2 vs 15.1 ( $\mathrm{p}<0.0001)$.

O ganho na habilidade de interpretação do eletrocardiograma em estudantes de medicina, comparando dois grupos, por Nilsson et al, o primeiro com intervenção assistido por software de computador com temas de princípios básicos em ECG, ECG patológicos e casos clínicos com eletrocardiograma por meio de textos, animações e imagens comparado ao ensino tradicional com aulas. Realizados pós testes (pontuação máxima 16) após período de 5 meses do estudo. O grupo intervenção obteve pontuação superior aos controles $(9,7$ vs 8,1$)$. Além disso, os pertencentes ao grupo intervenção foram questionados 
sobre apreensão do software em escala de 1 (ruim) a 5 (muito bom), classificando a utilidade em 4,1 e a qualidade do programa 3,9 .

Realizado um ensaio clínico randomizado por Davies et al com estudantes de medicina comparando em um grupo o uso de ferramenta digital de computador com ilustrações de princípios básicos de interpretação do eletrocardiograma comparado a outro grupo com ensino por meio de aulas tradicionais presenciais com apresentação no "Power Point" em duas sessões consecutivas de 30 minutos acerca dos princípios básicos do ECG. Os estudantes foram avaliados tanto no ganha de confiança (avaliado de 0 a 10) ao interpretar ECG quanto pós teste de conhecimentos. No pós teste o grupo tradicional obteve melhor atuação $(84 \%$ x 74,5\%). Para ganho de confiança ao interpretar o ECG a pontuação de ambos os grupos foram inicialmente 3/10 e após o ensino 7/10 e 8/10 (tradicional e ferramenta digital respectivamente).

Ainda para Davies et al o método tradicional levava a maior aquisição de conhecimento, mas que ambos aprimoravam a confiança do estudante na interpretação do ECG. A vantagem da ferramenta digital se daria no contexto de mais fácil disponibilização, revisão e atualização do material, além de poder ser acessado a qualquer momento. Dessa forma, é um método usado para complementar, e não substituir outras formas de ensino.

Bojsen et al avaliou o conhecimento pré teste e imediatamente pós teste, além disso, avaliação com teste de retenção em três grupos, curto prazo ( 2 a 4 semanas), médio prazo (10 a 12 semanas) e longo prazo (18 a 20 semanas) todos os testes com pontuação máxima de 100 pontos. Foi aplicado em estudantes de medicina com e sem conhecimentos prévios em cardiologia após realizar tutorial em um software com conhecimentos sobre eletrocardiograma e seus componentes, ritmo sinusal, causas de arritmias, bloqueios cardíacos, bloqueios de ramo, padrões de hipertrofia, eixo cardíaco, baixa voltagem e isquemia. Os estudantes com conhecimento prévio apresentaram médias maiores em todos os três testes. A média entre pré teste e pós teste foi de 52,7 e 68,4 respectivamente, porém com queda entre pós teste e testes de retenção para 59,9. Dessa forma, Bojsen et al evidenciou que houve queda no conhecimento de interpretação em ECG após semanas de ser realizado o tutorial, mas ainda em níveis maiores comparados ao pré teste e não houve diferença significativa na média do teste de retenção com o prazo que foi realizado (60.2, 60.8, 58.6 respectivamente). Assim, o software foi efetivo para o ensino do ECG em estudantes com níveis diferentes de conhecimento prévio e, apesar de declínio da retenção do aprendizado era ainda superior à antes do ensino. Provou que os softwares podem ser usados de forma isolada para ensino do ECG, mas não esclarece de que forma isto seria introduzido no currículo médico ou usado de forma para reforçar sob demanda o conhecimento seguido de intervenções baseadas em aulas tradicionais.

Keis et al analisou de forma qualitativa a opinião de estudantes de medicina sobre o ensino tradicional em aulas e o ensino por meio de curso digital para interpretação do ECG com exercícios de treinamento, sendo a próxima sessão liberada após conclusão da anterior. As principais desvantagens do método digital foi necessidade de maior iniciativa e disciplina própria, além da falta de interação. A principal vantagem se deu por poder realizar o método com tempo flexível quando desejado e quantas vezes fosse necessário, porém, o espaço e tempo fixos do modelo tradicional estimularia o estudante a participar e estudar o assunto. Os principais motivos que fizeram os estudantes a abandonarem o modelo digital foi a falta de motivação e pressão extrínseca, necessitando de mais iniciativa e disciplina por parte do estudante. Dessa forma, especificar um tempo limite no modelo digital e integrar ao ensino radical seria o recomendado.

Viljoen et al avaliou o ensino da interpretação em estudantes de medicina com conhecimentos prévios em ECG comparando dois grupos, o primeiro com modelo tradicional em aulas e o segundo com modelo combinado de aulas tradicionais associado a uso de software online de livre acesso para facilitar análise do ECG com feedback e analisado avaliação tanto quantitativa quanto qualitativa. O quantitativo se deu por aplicação do pré teste e pós teste após 6 semanas e 6 meses de ensino, o primeiro grupo (tradicional) teve aumento em 1.6 vezes (31.2 para 50.3) e o segundo grupo (combinado) aumento de 2.4 vezes (31 para 75.3) na média dos testes, aos 6 meses houve queda comparado com 6 semanas, mas, 
comparado ao início retenção superior de conhecimento para a estratégia combinada (37.6 e 57.7 respectivamente). Na análise qualitativa a vantagem seria permitir a prática e revisão da análise e interpretação do ECG, além de poder realizar de forma remota quando e onde fosse conveniente, a desvantagem seria relacionada ao próprio software já que não explicava após resultado correto ou incorreto, apresentava número limitado de exemplos de cada condição e presença de erros nas páginas digitais. Dessa forma, estratégia combinada resultou em melhor aquisição de competências na análise do ECG que a estratégias de aulas tradicionais isoladamente.

Fent et al submeteu estudantes de medicina e médicos recém-formados a, em um grupo, aulas tradicionais e, no segundo grupo, software de computador com interpretação de ECG e animações interativas em 3D e avaliou, de forma quantitativa e qualitativa, a atuação em interpretar ECG imediatamente após e 3 meses após a aula por meio de questionário. $\mathrm{Na}$ abordagem qualitativa não houve diferença quanto a ganho de confiança na interpretação do ECG quando comparado as duas estratégias, as principais vantagens eram que poderiam aprender no seu próprio ritmo, as animações ajudaram no aprendizado e a aparência era boa, as principais críticas foram que gostariam de ser possível realizar perguntas e que se tivessem mais áudios e vídeos em vez de explicações em texto aprimoraria melhor o software. Na abordagem quantitativa, as médias do grupo baseado em aulas foi superior ao baseado no software, embora não estatisticamente significante (7.07 vs 6.62, $\mathrm{p}=0.12$ ), envolvendo apenas os estudantes de medicina o grupo em aulas também foi superior ao grupo do software, mas também não estatisticamente significante (6.67 vs $6.28, \mathrm{p}=0.28$ ).

Kopéc et al comparou a efetividade entre dois métodos diferentes de ensino com aprendizado eletrônico e dividiu entre dois grupos estudantes de medicina, o primeiro colaborativo com grupo de 6 estudantes que respondiam às perguntas individualmente e após 24 horas em plataforma da internet discutiam em grupo sobre o assunto para uma resposta final e o segundo grupo individual com material educativo e respondiam aos testes isoladamente. Encontrou que o grupo colaborativo em que houve discussão em grupo apresentou média final superior aos que responderam isoladamente (6,4 e 5,6 respectivamente, $\mathrm{p}=0.04$ ).

Avaliando residentes no departamento de emergência Barthelemy et al comparou a acurácia do ensino na interpretação de ECG clinicamente significativos baseado em aulas tradicionais (dois tutores com duas apresentações de 180 minutos cada) com o ensino baseado em aprendizado eletrônico (uso de plataforma digital demonstrando princípios básicos de ECG e alterações) e avaliados em pós teste após 4 meses do término do curso composto de 10 questões e requerendo interpretação do ECG. Na avaliação pré teste a acurácia entre grupos foi de 37,5\% e 42,1\% respectivamente e, pós teste de $51 \%$ e $59,5 \%$ respectivamente $(\mathrm{p}=0.14)$. As condições com melhora significativa foram flutter atrial $(\mathrm{p}=0.044)$, bloqueios de ramos $(\mathrm{p}=0.007)$ e infarto do miocárdio $(\mathrm{p}=0.005)$ e que não obteve aprimoramento foi detecção de eletrodos periféricos mal posicionados $(\mathrm{p}=0.5)$.

\section{Conclusão}

Considerando os estudos analisados, o uso softwares de computadores e de outros dispositivos eletrônicos tiveram crescimento cada vez maior nas últimas décadas e tiveram potencial para prover aprimoramento nas habilidades de interpretação do eletrocardiograma, uma vez que esse permite disponibilidade em horários e locais mais flexíveis, repetição dos assuntos o quanto for necessário e atualização do conteúdo periodicamente. Contudo, a não definição de um horário específico para ensino, a necessidade de disciplina por parte isolada do aprendiz, a ausência de interatividade em grupo são fatores que distanciam, de certa forma, os estudantes desse método, mostrando que, esse ensino não deve ser usado de forma isolada, mas sim, de forma a complementar o método tradicional de ensino baseado em aulas. 
Dessa forma, com as ressalvas aqui citadas, estudos futuros com o desenvolvimento de novos aplicativos e softwares digitais com maior adesão, controle, aplicabilidade e interação sejam realizados, visando comparação entre as três estratégias de ensino (tradicional, digital e mista) para que possam trazer evidências científicas confiáveis para implantação efetiva destas ferramentas de ensino.

\section{Referências}

Akbarzadeh, F., Kazemi, B., Alizadeh, A., \& Akbarzadeh, A. (2012). The Efficacy of Web-Based Multimedia Education of Normal Electrocardiogram in Junior and Senior Medical Students. Research and Development in Medical Education. 1. 10.5681/rdme.2012.016.

Barthelemy, F. X., Segard, J., Fradin, P., Hourdin, N., Batard, E., Pottier, P., Potel, G., \& Montassier, E. (2017). ECG interpretation in Emergency Department residents: an update and e-learning as a resource to improve skills. Eur J Emerg Med.

Bojsen, S., Räder, S. Holst, A., Kayser, L., Ringsted, C., Svendsen, J., \& Konge, L. (2015). The acquisition and retention of ECG interpretation skills after a standardized web-based ECG tutorial-a randomised study. BMC medical education.

Cantillon, P. (2003). ABC of learning and teaching in medicine: Teaching large groups. BMJ;7386(326):437-437.

Clayden, G. S., \& Wilson. B. (1988). Computer-assisted learning in medical education. Med Educ.

Davies, A., Macleod, R., Bennett-Britton, I., McElnay, P., Bakhbakhi, D., \& Sansom, J. (2016). E-learning and near-peer teaching in electrocardiogram education: a randomised trial. Clin Teach.

Fent, G., Gosai, J., \& Purva, M. (2015). Teaching the interpretation of electrocardiograms: which method is best? J Electrocardiol.

Fent, G., Gosai, J., \& Purva, M. (2016). A randomized control trial comparing use of a novel electrocardiogram simulator with traditional teaching in the acquisition of electrocardiogram interpretation skill. JournalofElectrocardiology.

Fincher, R. E., Abdulla, A. M., Sridharan, M. R., Houghton, J. L., \& Henke, J. S. (19888). Computer-assisted learning compared with weekly seminars for teaching fundamental electrocardiography to junior medical students. South Med J.

Giffoni, R. T., \& Torres, R. M. (2010). Breve história da eletrocardiografia. Revista Médica de Minas Gerais; 20(2):263-270.

Henry, J. B. (1990). Computers in medical education: information and knowledge management, understanding, and learning. Hum Pathol.

Keis, O., Grab, C., Schneider, A., \& Öchsner, W. (2017). Online or face-to-face instruction? A qualitative study on the electrocardiogram course at the University of Ulm to examine why students choose a particular format. BMC Medical Education.

Kopeć, G., Waligóra, M., Pacia, M., Chmielak, W., Stępień, A., Janiec, S., Magoń, W., Jonas, K., \& Podolec, P. (2018). Electrocardiogram reading: a randomized study comparing 2 e-learning methods for medical students. Pol Arch Intern Med.

Marçal, E., Andrade, R., \& Rios, R. (2005). Aprendizagem utilizando Dispositivos Móveis com Sistemas de Realidade Virtual. RENOTE RevistaNovasTecnologiasnaEducação.

Montassier, E., Hardouin, J. B., Segard, J., Batard, E., Potel, G., Planchon, B., et al. (2016). e-Learning versus lecture-based courses in ECG interpretation for undergraduate medical students: a randomized noninferiority study. Eur J Emerg Med

Nilsson, M., Bolinder, G., Held, C., Johansson, B., Fors, U., \& Östergren, J. (2008). Evaluation of a web-based ECG-interpretation programme for undergraduate medical students. BMC Medical Education2008; 8:25.

Pastore, C. A., Pinho, J. A., Pinho, C., Samesima, N., Pereira Filho, H. G., Kruse, J. C. L., et al. (2016). III Diretrizes da Sociedade Brasileira de Cardiologia Sobre Análise e Emissão de Laudos Eletrocardiográficos. ArqBrasCardiol; 106(4)1:1-23.

Piemme, T. E. (1988). Computer-assisted learning and evaluation in medicine. JAMA

Pontes, P. A. I., Chaves, R. O., Castro, R. C., de Souza, É. F., Seruffo, M. C. R., \& Francês, C. R. L. (2018). Educational Software Applied in Teaching Electrocardiogram: A Systematic Review. Biomed Res Int.

Sattar, Y., \& Chhabra, L. (2021). Electrocardiogram. In: StatPearls. Treasure Island (FL): StatPearls Publishing; https://www.ncbi.nlm.ni h.gov/books/NBK549803/

Viljoen, C. A., Millar, R. S., Manning, K., \& Burch, V. C. (2020). Effectiveness of blended learning versus lectures alone on ECG analysis and interpretation by medical students. BMC medical education, 20(1), 488. 